\title{
Spectral and temporal control of Q-switched solid-state lasers using intracavity MEMS
}

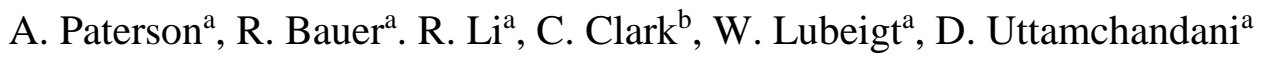 \\ ${ }^{a}$ University of Strathclyde, Dept. of Electronic and Electrical Engineering, Centre for Microsystems \\ and Photonics, TIC Level 5, 99 George Street, Glasgow, G1 1RD, UK \\ ${ }^{\mathrm{b}}$ Helia Photonics, Rosebank Park, Livingston, EH54 7EJ, UK
}

\begin{abstract}
Active control of the spectral and temporal output characteristics of solid-state lasers through use of MEMS scanning micromirrors is presented. A side-pumped Nd:YAG laser with two intracavity scanning micromirrors, enabling Qswitching operation with controllable pulse duration and pulse-on-demand capabilities, is investigated. Changing the actuation signal of one micromirror allows a variation of the pulse duration between $370 \mathrm{~ns}$ and $1.06 \mu \mathrm{s}$ at a pulse repetition frequency of $21.37 \mathrm{kHz}$ and average output power of $50 \mathrm{~mW}$. Pulse-on-demand lasing is enabled through actuation of the second micromirror. To our knowledge this is the first demonstration of the use of multiple intracavity MEMS devices as active tuning elements in a single solid-state laser cavity. Furthermore, we present the first demonstration of control over the output wavelength of a solid-state laser using a micromirror and a prism in an intracavity Littman configuration. A static tilt actuation of the micromirror resulted in tuning the output wavelength of an $\mathrm{Yb}: \mathrm{KGW}$ laser from $1024 \mathrm{~nm}$ to $1031.5 \mathrm{~nm}$, with FWHM bandwidths between $0.2 \mathrm{~nm}$ and $0.4 \mathrm{~nm}$. These proof-ofprinciple demonstrations provide the first steps towards a miniaturized, flexible solid-state laser system with potential defense and industrial applications.
\end{abstract}

Keywords: Solid-state laser, Q-switch, wavelength tuning, MEMS, scanning micromirror

\section{INTRODUCTION}

The use of micro-electro-mechanical systems (MEMS) as active elements in laser systems gives the opportunity for miniaturized alternatives to bulk optics devices, having lower electrical power demands, lower cost due to batch fabrication and the possibility of added functionality. Some of the most common applications include MEMS laser scanners [1] and MEMS-tunable VCSELs [2]. MEMS Q-switching has also been demonstrated in fiber lasers [3], solidstate lasers [4,5] and a microchip laser [6] using cantilever- and micromirror-type devices. Added functionality through the use of MEMS elements was, for example, achieved by creating multiple, individually controllable laser beams from a single laser gain medium [7].

This paper presents our initial investigation on the use of MEMS scanning micromirrors to actively tune the temporal and spectral output characteristics of solid-state lasers while simultaneously serving as intracavity mirrors. Two micromirror actuation techniques are used in this work: electrostatically actuated micromirrors for resonant scanning at $\sim 10 \mathrm{kHz}$; and electrothermally actuated micromirrors for quasi-static angular displacement. The electrostatically actuated micromirrors are optically coated with dielectric layer pairs $\left(\mathrm{SiO}_{2} / \mathrm{Nb}_{2} \mathrm{O}_{5}\right)$ to enhance the reflectivity at $\lambda=1064 \mathrm{~nm}$, while the electrothermally actuated micromirrors are gold-coated. Temporal tuning of the output of a Q-switched $\mathrm{Nd}$ :YAG laser platform is demonstrated using both MEMS designs inserted in a single cavity. In this arrangement, the electrostatically actuated micromirror functions as an active Q-switch element with variable speed to tune the output pulse duration, while the electrothermally actuated micromirror functions as an on/off switch enabling pulse-on-demand capability. Furthermore, spectral tuning of the output of an $\mathrm{Yb}: \mathrm{KGW}$ laser platform is demonstrated using an electrothermally actuated micromirror and a bulk prism in a Littman configuration.

Finally, potential improvements to the demonstrated techniques are discussed, highlighting the key areas for future investigation. The individual demonstrations presented in this paper provide a platform for combining the spectral and temporal tuning techniques, which would allow for a miniaturized solid-state laser with controllable spectral and temporal output characteristics. 


\section{METHODOLOGY}

\subsection{MEMS fabrication, design and optical coatings}

The MEMS used in this work are scanning micromirrors which were fabricated using a silicon-on-insulator multi-user MEMS process (SOIMUMPs) provided by MEMSCAP Inc. [8]. Starting with a $400 \mu \mathrm{m}$ thick silicon substrate and a $1 \mu \mathrm{m}$ thick layer of buried oxide for electrical isolation, either a $10 \mu \mathrm{m}$ or $25 \mu \mathrm{m}$ thick device layer of phosphorousdoped, single-crystal-silicon is added. This device layer is patterned to the customer design by a deep reactive ion etch step down to the oxide layer. A further deep reactive ion etch step on the silicon substrate and a wet etch step on the oxide layer then fully releases the patterned device layer, producing the moving parts which form a functional micromirror. A masked pad-metal process deposits a gold layer for the electrical bond pads and connection routes, with a thin inter-layer of chromium for adhesion.

Two separate principles of micromirror actuation are used in this work: electrostatic (ES) and electrothermal (ET) actuation. Our ES circular micromirror design $(750 \mu \mathrm{m}$ in diameter) uses the $25 \mu \mathrm{m}$ thick single-crystal-silicon device layer and operates via a single-sided, angled vertical-offset comb-drive. The comb-drive is located $200 \mu \mathrm{m}$ from the micromirror edge on one of the two suspension springs connecting the micromirror to the silicon substrate. It consists of 12 pairs of interleaved moving and fixed combs of length $160 \mu \mathrm{m}$ and width $10 \mu \mathrm{m}$ with lateral comb gaps of $6 \mu \mathrm{m}$. The suspension springs are each $750 \mu \mathrm{m}$ long and $17 \mu \mathrm{m}$ wide. The structural geometry, shown in Figure 1(a), was investigated using the FEM software COMSOL Multiphysics to obtain the mechanical eigenfrequencies for different dynamic modes. The anisotropic Young's modulus properties of silicon [9], and a [110] axis orientation were considered to coincide with MEMSCAP Inc.'s single-crystal-silicon wafer growth properties. The desired torsional resonance mode, also shown in Figure 1(a), was simulated to be at $10.92 \mathrm{kHz}$ with a minimum gap of $1.5 \mathrm{kHz}$ to any parasitic, in-plane movement modes.

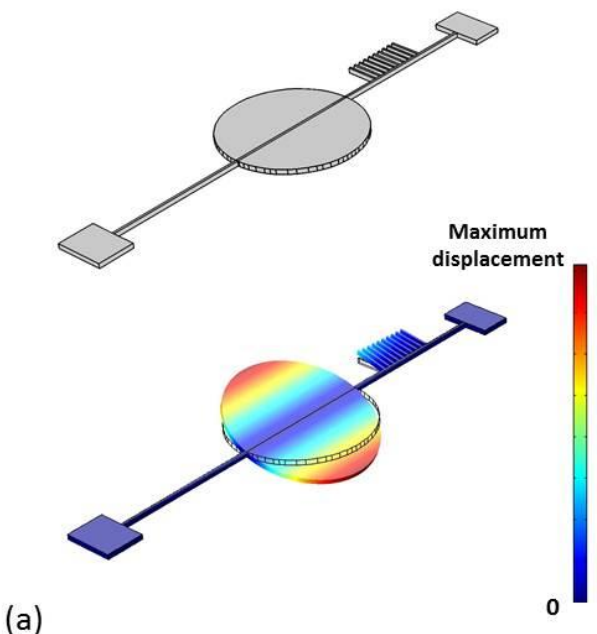

(a)

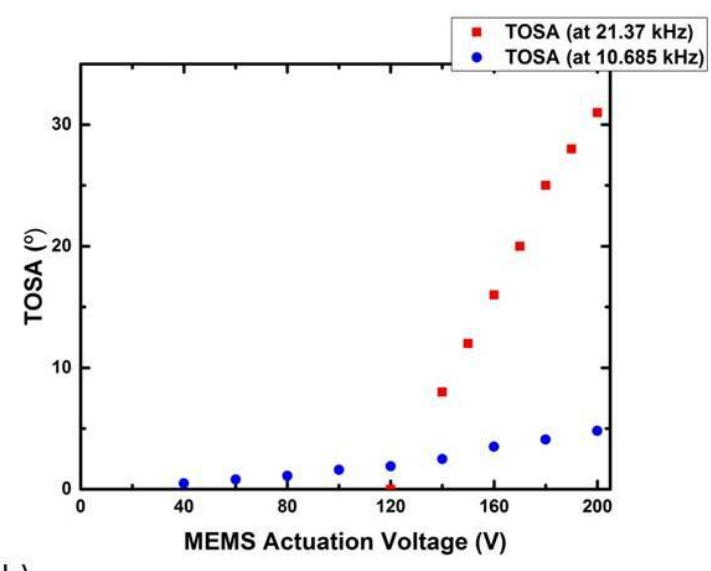

(b)

Figure 1. (a) FEM simulated images of the ES micromirror geometry and torsional movement mode, (b) The total optical scanning angle (TOSA) of the micromirror as a function of MEMS actuation voltage and frequency.

To maintain efficient use as an intracavity laser element a multilayer dielectric coating was deposited on the ES micromirror surface using ion-assisted electron beam deposition (provided by Helia Photonics Ltd). The coating comprised 13 layer pairs of $\lambda / 4$ thick $\mathrm{SiO}_{2} / \mathrm{Nb}_{2} \mathrm{O}_{5}$, resulting in a reflectivity of $>98 \%$ at $\lambda=1064 \mathrm{~nm}$. An induced change in radius of curvature (ROC) of the micromirror surface was observed, caused by stresses between the device layer and the dielectric coating layers due to their different thermal expansion coefficients. The ROC was measured to be $60 \mathrm{~mm}$ convex, after coating, using a white light interferometer. Experimental analysis of the micromirror motion behavior was performed by applying a square-wave voltage signal to the fixed combs of the actuator using a signal generator and an amplifier. The torsional resonance movement occurred at a frequency of $10.685 \mathrm{kHz}$ and its $2 \mathrm{f}$ harmonic $21.37 \mathrm{kHz}$, as shown in Figure 1(b). Driven at $10.685 \mathrm{kHz}$, the total optical scan angle (TOSA) of the micromirror varied from $0.5^{\circ}$ to $4.8^{\circ}$ as the actuation voltage varied from $40 \mathrm{~V}$ to $200 \mathrm{~V}$. Driven at $21.37 \mathrm{kHz}$, the TOSA of the micromirror varied from 
$8.0^{\circ}$ to $33^{\circ}$ as the actuation voltage varied from $140 \mathrm{~V}$ to $200 \mathrm{~V}$. The mechanical oscillation frequency of the micromirror was $10.685 \mathrm{kHz}$ for both the resonant and $2 \mathrm{f}$ harmonic actuation schemes.

Our ET circular micromirror design, shown in Figure 2(a), uses the $10 \mu \mathrm{m}$ thick single-crystal-silicon device layer and operates via Joule heating and mechanical constraints. Four actuators, consisting of three longitudinal beams $(50 \mu \mathrm{m}$ wide, $1800 \mu \mathrm{m}$ long, $150 \mu \mathrm{m}$ between) constrained at one end by a connecting beam ( $60 \mu \mathrm{m}$ wide, $450 \mu \mathrm{m}$ long), are driven by applying a voltage signal to the two outer longitudinal beams. The resulting electrical current will cause Joule heating and therefore thermal expansion of the actuated beams, while the electrically isolated, central beam acts as a constraint to force an out-of-plane movement of the microactuator to tilt the micromirror. The four radially positioned actuators enable 2D control of the micromirror tilt angle. COMSOL Multiphysics was used to simulate the micromirror movement during electrical actuation, which is shown in Figure 2(b). Two variations of this design were used in this work with the only difference being the micromirror diameters $(1.4 \mathrm{~mm}$ and $2 \mathrm{~mm})$. To increase the surface reflectivity a $200 \mathrm{~nm}$ thick gold coating was deposited on the micromirror surfaces using thermal evaporation. The measured reflectivity was $96 \%$ at $\lambda \sim 1 \mu \mathrm{m}$ and the measured ROC was $50 \mathrm{~mm}$ concave for the $1.4 \mathrm{~mm}$ diameter device and $75 \mathrm{~mm}$ concave for the $2 \mathrm{~mm}$ diameter device. The micromirror movement was evaluated by applying a D.C. voltage to one of the device actuators using a D.C. power supply and LabVIEW for control. The $1.4 \mathrm{~mm}$ diameter micromirror exhibited a range of tilt angles from $0^{\circ}$ to $2.3^{\circ}$ for actuation voltages from $0 \mathrm{~V}$ to $11.4 \mathrm{~V}$. The $2 \mathrm{~mm}$ diameter micromirror exhibited a range of tilt angles from $0^{\circ}$ to $1.2^{\circ}$ for actuation voltages from $0 \mathrm{~V}$ to $11.4 \mathrm{~V}$. The movement threshold was $\sim 4 \mathrm{~V}$ for both devices with the maximum voltage limited to below $12 \mathrm{~V}$ to avoid thermal damage of the micromirrors. The actuation was applicable in both directions from the rest position.

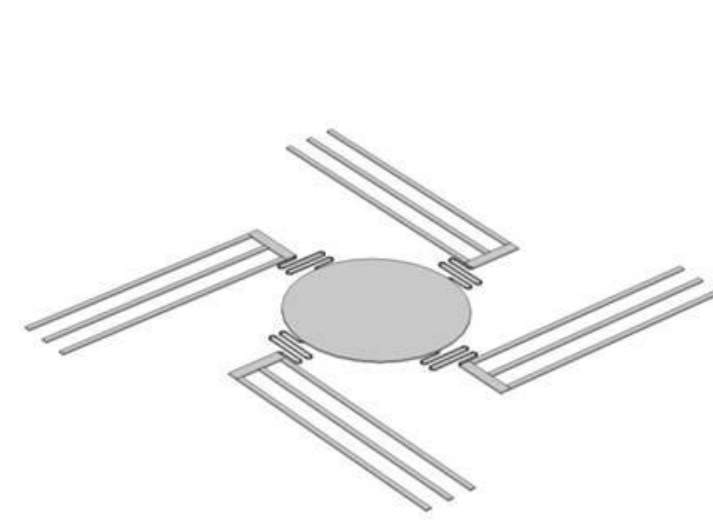

(a)

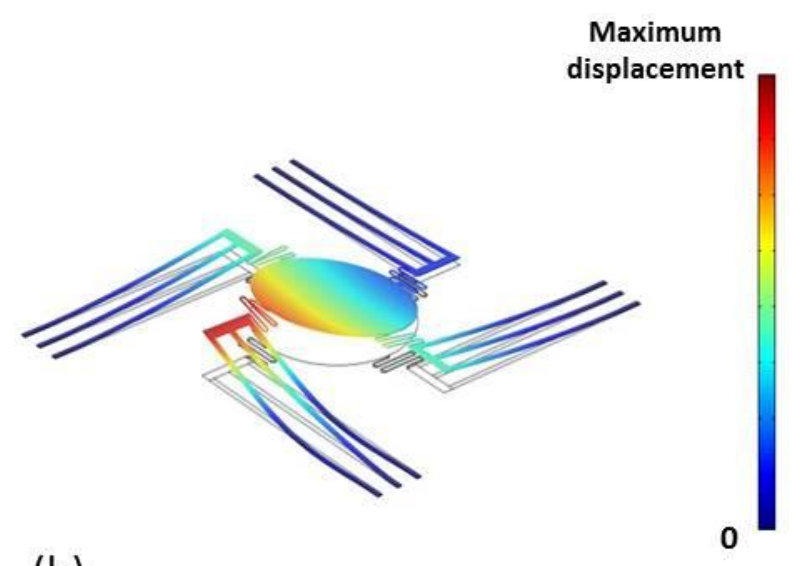

(b)

Figure 2. (a) FEM simulated image of the ET micromirror geometry, (b) FEM simulated image of the ET micromirror tilt movement as current is applied to one of the actuators.

\subsection{Temporal control of the laser output}

When an ES micromirror is actuated at its resonance point, laser Q-switching is enabled when the micromirror surface normal resonantly scans through cavity alignment. This will produce a train of output pulses at two times the mechanical oscillation frequency of the micromirror, due to the bi-directional scanning movement [5]. The work in this paper extends this technique to investigate variable pulse duration and on-demand pulse bursts through implementing multiple MEMS devices into a single laser cavity. The technique is illustrated in Figure 3.

Variation of the pulse duration can be achieved through controlling the speed at which the intracavity ES micromirror resonantly scans through cavity alignment. One method of doing this is to vary the amplitude of the actuation voltage to the MEMS, inherently changing the scanning angle of the micromirror. The larger the scanning angle the faster the micromirror scans through cavity alignment and thus the shorter the laser output pulse duration. Building on this an additional ET micromirror in the same laser cavity can provide an on-off shutter through tilting in and out of cavity alignment, enabling pulse-on-demand and bursts of pulses at a specified frequency. These principles have been investigated and are described in section 3. 


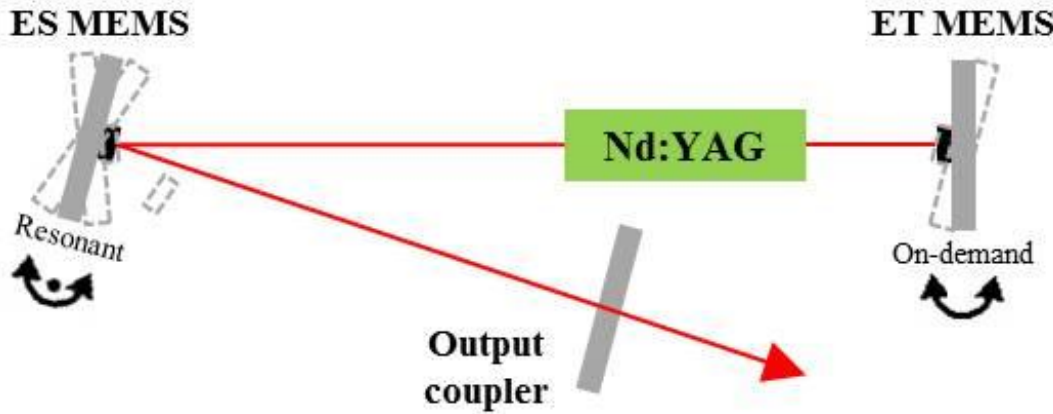

Figure 3. Sample schematic of a Q-switched laser cavity with two MEMS devices.

\subsection{Spectral control of the laser output}

A gain medium with a large gain bandwidth, e.g. $\sim 40 \mathrm{~nm}$ theoretical bandwidth in $\mathrm{Yb}: \mathrm{KGW}$, makes it possible to tune the output lasing wavelength using a wavelength dispersive element and a rotating mirror in a Littman configuration [10]. Figure 4 shows an example layout using a prism as the wavelength dispersing element and an ET micromirror as the wavelength selecting, rotating mirror. Static actuation of the intracavity ET micromirror provides the tilt required to angularly select the feedback for the lasing wavelengths dispersed by the prism, which is inserted close to the Brewster angle for the center wavelength of the gain bandwidth $(1040 \mathrm{~nm})$. The reflected light from the prism surface on the micromirror side of the cavity is used as the laser output. This configuration is described in greater detail in section 4.

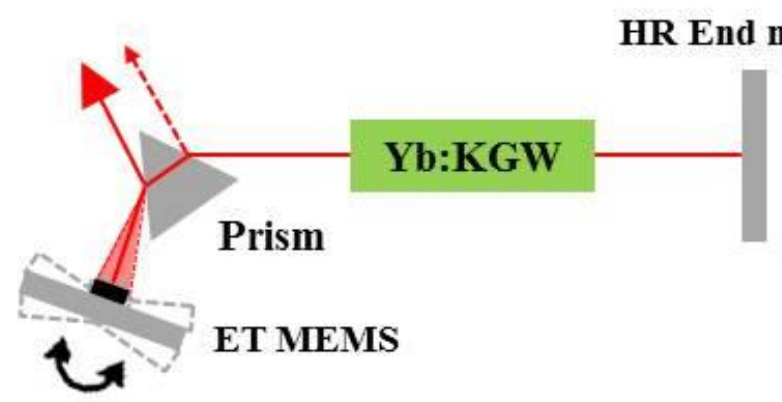

Figure 4. Sample schematic of a laser cavity for wavelength tuning.

\section{TEMPORAL CONTROL OF A Nd:YAG LASER}

\subsection{Laser cavity design}

A five mirror cavity was designed to investigate temporal Q-switch tuning, shown in Figure 5, incorporating two MEMS devices: one ES micromirror and one ET micromirror. The cavity was built around a Northrup Grumman Nd:YAG laser module, which consisted of a Nd:YAG laser rod (63 mm long, $3 \mathrm{~mm}$ in diameter) side-pumped radially at $808 \mathrm{~nm}$ by three stacks of laser diodes. On one side of the gain medium a high-reflective (HR) folding mirror with ROC $=100 \mathrm{~mm}$ is inserted before the ES MEMS end mirror with ROC $=-60 \mathrm{~mm}$ (convex). On the other side of the Nd:YAG module is the ET micromirror with ROC $=50 \mathrm{~mm}$, a further HR folding mirror with $\mathrm{ROC}=100 \mathrm{~mm}$ and an $\mathrm{R}=80 \%$ flat output coupler to complete the cavity. The overall cavity length is $480 \mathrm{~mm}$. The cavity was designed to accommodate the limitations of the MEMS devices, notably their optical surface diameters and their surface curvature sensitivity to excessive temperature changes. Since the micromirrors are $<99 \%$ reflective a significant level of absorption occurs within the material layers of the device. Non-reflected laser light will cause the micromirror to heat up and change curvature due to the difference in thermal expansion coefficients between the device layers. Therefore, to enable powerscaling the cavity must be designed to be stable for a large range of curvatures at the locations of the micromirrors. 


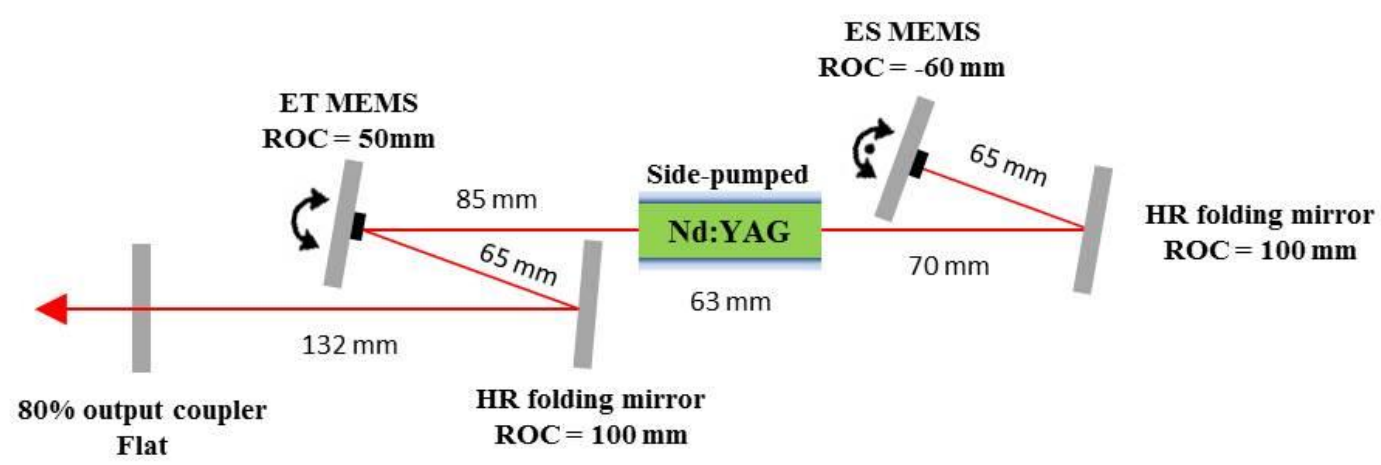

Figure 5. The designed five mirror laser cavity, incorporating two MEMS micromirrors, used to investigate variable Qswitch pulse duration and pulse-on-demand.

\subsection{Laser Q-switching with variable pulse duration}

Q-switching was generated through actuation of the ES micromirror, achieved by applying a square-wave voltage signal at $21.37 \mathrm{kHz}$ to the comb-drive. The corresponding resonant movement frequency of the micromirror was $10.685 \mathrm{kHz}$. The temporal Q-switched laser output was measured by focusing it onto a $1.5 \mathrm{GHz}$ photodiode with the laser tuned to $50 \mathrm{~mW}$ of average output power for each measurement. This average output power was intentionally limited to prevent an excessive heat-induced curvature change of the micromirror. The MEMS actuation voltage was varied from $200 \mathrm{~V}$ to $140 \mathrm{~V}$ in intermediate steps of $10 \mathrm{~V}$, corresponding to an overall change in micromirror TOSA from $31^{\circ}$ to $8^{\circ}$ respectively. The change in micromirror TOSA, and inherently the rotational speed of the micromirror scanning through cavity alignment, resulted in a variation of the full width half maximum (FWHM) pulse duration from $370 \mathrm{~ns}$ to $1.06 \mu \mathrm{s}$, respectively, at a constant pulse repetition frequency (PRF) of $21.37 \mathrm{kHz}$. At $50 \mathrm{~mW}$ this corresponds to a change from $5.9 \mathrm{~W}$ to $2.1 \mathrm{~W}$ in pulse peak power respectively. The PRF is double the movement frequency of the micromirror since the micromirror surface normal is scanned twice through cavity alignment during a single movement cycle. The variation of pulse durations is shown in Figure 6 alongside the output beam profiles at $200 \mathrm{~V}$ and $140 \mathrm{~V}$ MEMS actuation. The observed double output spot is caused by a non-optimum pulse build-up time, resulting in a time delay between the micromirror scanning through its midpoint and pulse emission [5]. Since pulse emission occurs at both movement directions from the midpoint, a spatial offset is present between two consecutive pulses. This spatial offset reduces with the rotational speed of the micromirror as it passes through cavity alignment. The measured $\mathrm{M}^{2}$ values for each spatially offset beam at both $200 \mathrm{~V}$ and $140 \mathrm{~V}$ MEMS actuation were $\leq 1.2$.

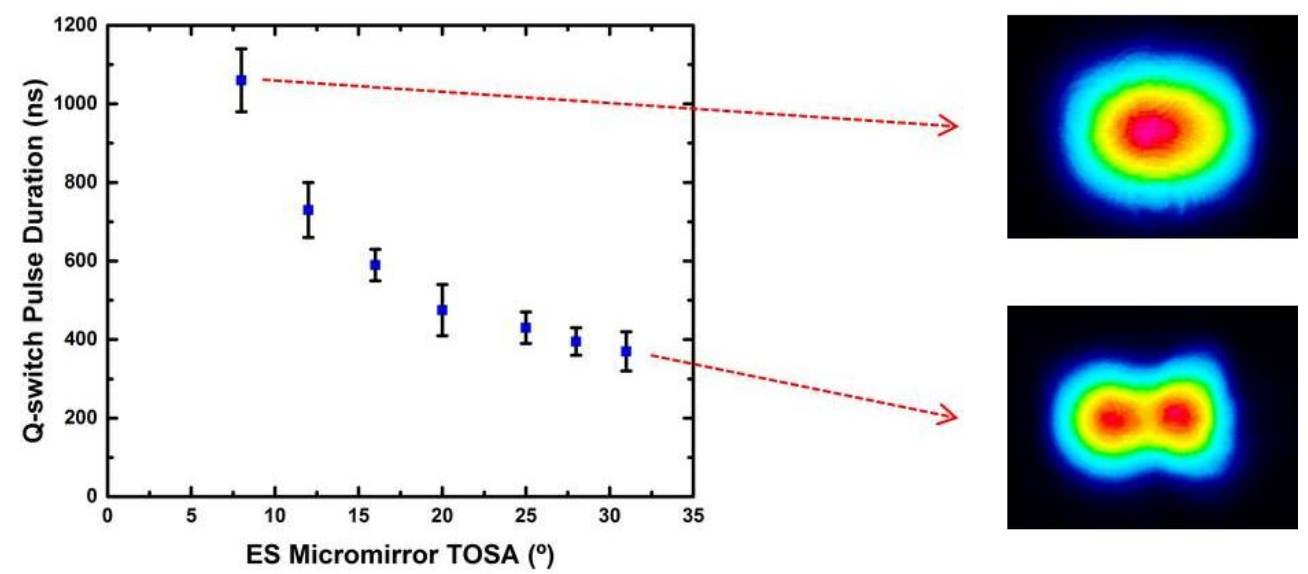

Figure 6. Plot of laser Q-switch pulse duration v TOSA with output beam profiles at the two extremes. 


\subsection{Pulse-on-demand capability}

The output state of the laser (on or off) can be selected on-demand during Q-switch operation by simultaneously applying a voltage signal to the actuators of the ET micromirror, causing a static angular tilt in or out of cavity alignment. Using a signal generator a $10 \mathrm{~V}$ square-wave signal was applied to one of the four actuators of the ET micromirror, with the frequency of the square-wave determining the on-off status of the Q-switched laser output. A $10 \mathrm{~Hz}$ signal was investigated with the resulting pulse train shown in Figure 7(a), again at an average output power of $50 \mathrm{~mW}$ (on-time only). The ET micromirror had a switching time of $5 \mathrm{~ms}$ and a successive movement overshoot period of $\sim 10 \mathrm{~ms}$ was observed before the micromirror came to rest, as shown in Figure 7(b).

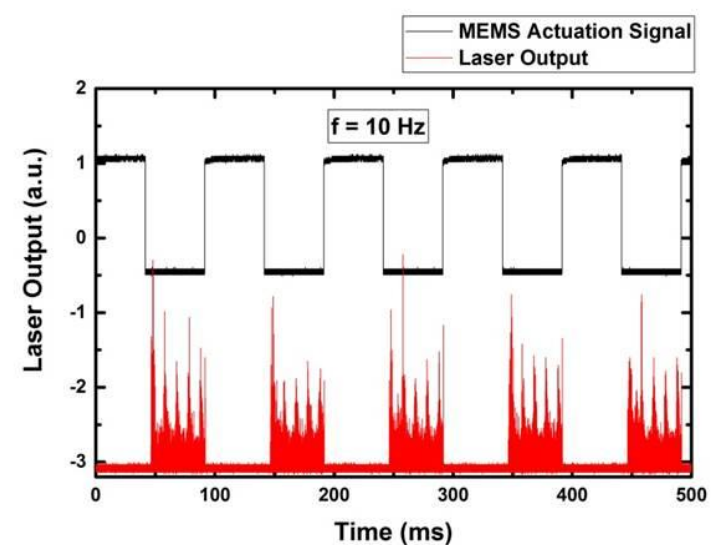

(a)

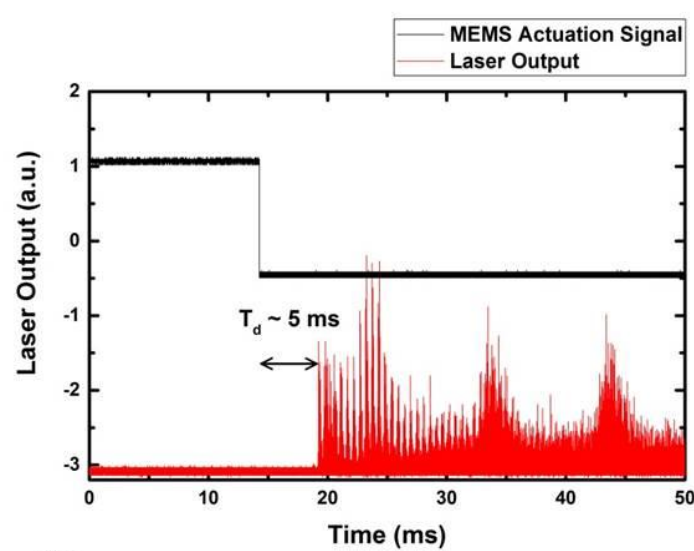

(b)

Figure 7. (a) $10 \mathrm{~Hz}$ trains of pulses obtained by applying a $10 \mathrm{~Hz}$ voltage signal to the actuator of the ET micromirror, (b) A close-up view of the start of one of the pulse trains.

\subsection{Discussion}

The limitations of this initial investigation occur primarily in the MEMS optical coatings and the power supply to the laser pump diodes. Power-scaling is limited by the thermally induced curvature of the micromirrors, stemming from absorption of non-reflected laser light within the device layers. Improvements on the HR coatings (desired reflectivity $\mathrm{R}>99.9 \%$ ) with less curvature sensitivity to temperature would enable longer pulse durations (covering the entire range of achievable micromirror TOSAs) and higher average output powers. A further hindrance of the power-scaling limitations is a parasitic $100 \mathrm{~Hz}$ current ripple present in the output signal from the power supply to the laser pump diodes, which can be observed in Figure 7. Working close to laser threshold, as present in the described work, means the current ripple has a significant impact on the output stability of the pulsed laser, particularly at longer pulse durations which have lower amplitude. Finally, the switch-on time and resonant movement of the ET micromirror could potentially be reduced using an appropriate PID control or a tailored waveform as the electrical driving signal for the ET actuator.

\section{SPECTRAL CONTROL OF A Yb:KGW LASER}

\subsection{Laser cavity design}

A three mirror cavity was designed, shown in Figure 8, to investigate intracavity wavelength tuning of a solid-state laser using a MEMS micromirror. The cavity was built around a right-angle cut $\mathrm{Yb}: \mathrm{KGW}$ crystal from Altechna (1\% $\mathrm{Yb}$ concentration). The crystal is $10 \mathrm{~mm}$ long with $5 \times 2 \mathrm{~mm}$ end facets and is anti-reflection coated for $\lambda=1030-1070 \mathrm{~nm}$ on each face. The gain medium is optically end-pumped by a $981 \mathrm{~nm}$ fiber-coupled diode laser and a lens imaging system, producing a $\sim 280 \mu \mathrm{m}$ diameter, collimated beam along the length of the crystal. On the pump side of the crystal is a $1 / 2$ " diameter, HR coated end mirror for $\lambda=1030-1200 \mathrm{~nm}$ with $\mathrm{ROC}=250 \mathrm{~mm}$. The end mirror is AR coated on the pump side between $808 \mathrm{~nm}$ and $980 \mathrm{~nm}$ to allow transmission of the pump light to the gain medium. Completing the cavity on the opposite side is a folding mirror with $\mathrm{ROC}=250 \mathrm{~mm}$, followed by an equilateral dispersive prism and an ET micromirror in a Littman configuration. The laser output is the reflected laser light from the prism surface on the 
micromirror side of the cavity. To quantify the output coupling from the prism the folding mirror is $98 \%$ reflective at $1060 \mathrm{~nm}$. The cavity dimensions were selected to match the intracavity laser field diameter with the pump laser beam diameter through the crystal and to ensure cavity stability over a range of micromirror surface curvatures from ROC $=$ $30 \mathrm{~nm}$ to a flat surface.

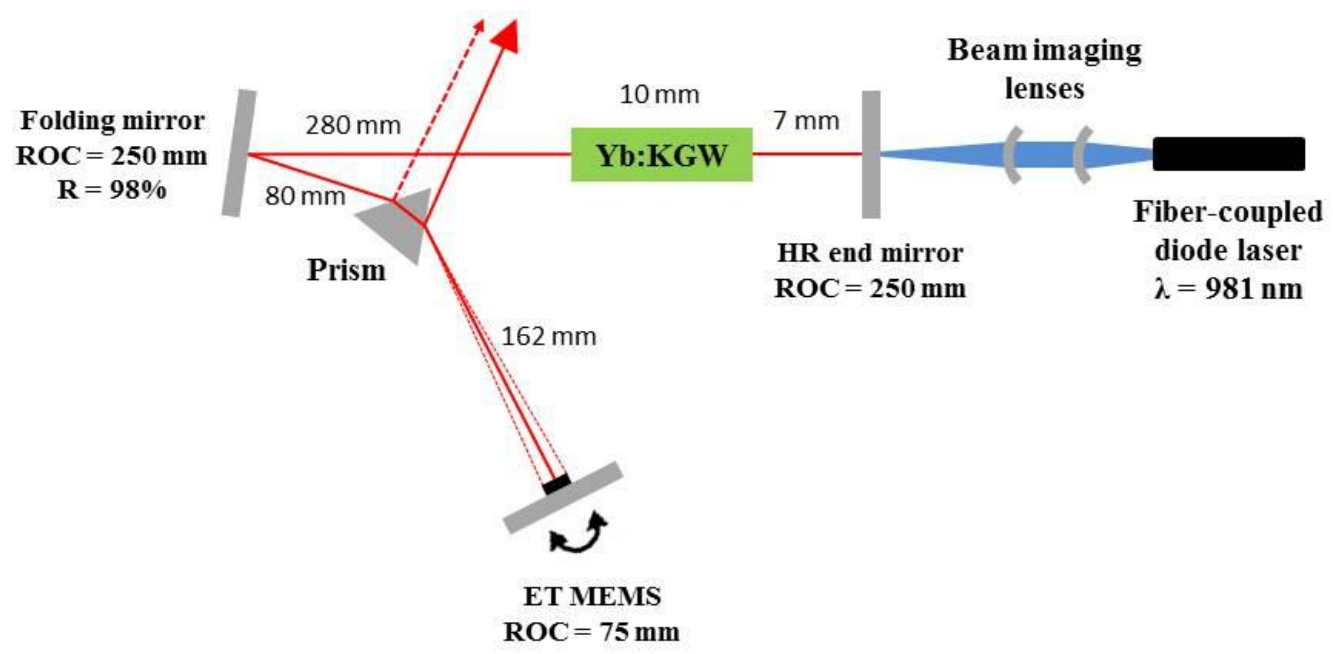

Figure 8. The three mirror laser cavity used to investigate wavelength tuning using an ET micromirror and a prism in a Littman configuration.

\subsection{Wavelength tuning by an intracavity micromirror}

Using the cavity in Figure 8, the wavelength of the laser output was measured using an optical spectrum analyzer as the ET micromirror was actuated. With no electrical actuation of the ET micromirror, the laser output peak wavelength occurred at $\lambda=1027 \mathrm{~nm}$ with an output power of $25 \mathrm{~mW}$ from one of the two outputs of the $\mathrm{R}=98 \%$ folding mirror. The corresponding output power from the prism was $<1 \mathrm{~mW}$, implying a very low output coupling. The wavelength was tunable to a minimum of $1024 \mathrm{~nm}$ and a maximum of $1031.5 \mathrm{~nm}$ as the micromirror was rotated by $\pm 0.1^{\circ}$ through electrical actuation, giving a total tuning range of $7.5 \mathrm{~nm}$ for this cavity configuration (see Figure 9). The output powers from the $\mathrm{R}=2 \%$ folding mirror at these wavelengths were $20 \mathrm{~mW}$ and $4 \mathrm{~mW}$ respectively. The FWHM linewidths are $0.4 \mathrm{~nm}$ at $\lambda=1024 \mathrm{~nm}, 0.4 \mathrm{~nm}$ at $\lambda=1027 \mathrm{~nm}$ and $0.2 \mathrm{~nm}$ at $\lambda=1031.5 \mathrm{~nm}$.

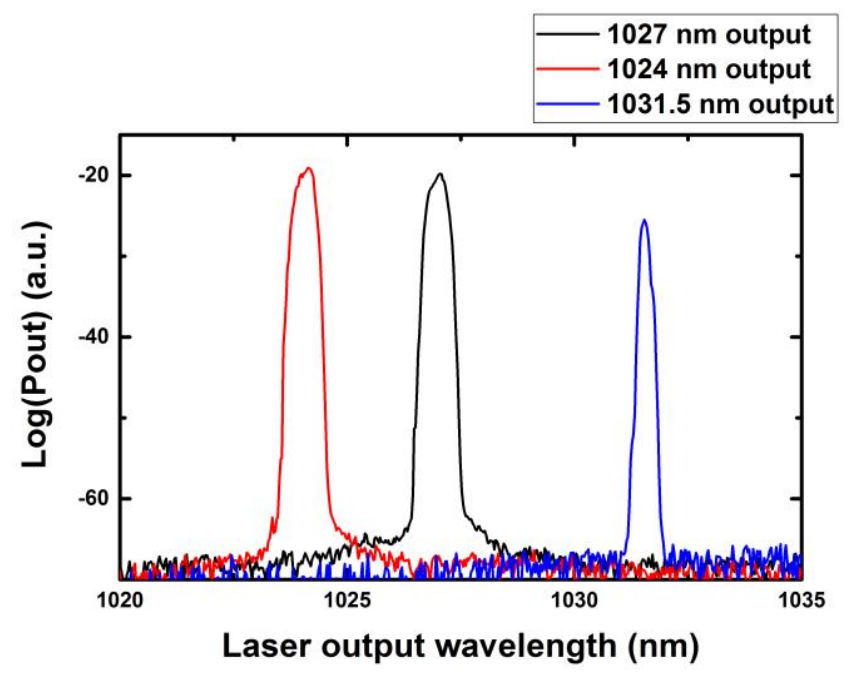

Figure 9. The laser output spectra for different ET micromirror actuation conditions. 


\subsection{Discussion}

This initial investigation demonstrated the proof-of-principle of wavelength tuning using this intracavity MEMS-based technique. Again power-scaling was limited by the heat-induced curvature change of the micromirror surface, meaning this technique would benefit from improved HR coatings. This initial demonstration was conducted with a nonoptimized cavity, with further investigation currently undertaken on such aspects as the effect of the distance between the prism and the rotating micromirror, and the influence of the prism output coupling through variation of the prism angular alignment.

During the process of laser alignment the folding mirror, prism and ET micromirror are placed in an extracavity configuration, providing feedback to a short 2-mirror alignment cavity. In the extracavity feedback configuration the wavelength was tunable from $1023 \mathrm{~nm}$ to $1045 \mathrm{~nm}$ through the ET micromirror actuation, with FWHM linewidths of $\sim 2 \mathrm{~nm}$. An investigation into the reduction of the tuning range when switching to an intracavity configuration will also be undertaken.

\section{CONCLUSION}

The use of MEMS scanning micromirrors as intracavity tuning elements for the temporal and spectral characteristics of solid-state lasers has been demonstrated. The micromirrors, coated with gold or dielectric layers, are used to control the temporal behavior of a Q-switched Nd:YAG laser and the spectral behavior of an Yb:KGW laser. Temporal control of the Nd:YAG laser was investigated using two micromirror designs as tuning elements. The first enabled laser Qswitching with tunable pulse duration between $370 \mathrm{~ns}$ and $1.06 \mu \mathrm{s}$, whilst the second could tilt in and out of laser alignment enabling pulse-on-demand lasing. Spectral control of the $\mathrm{Yb}: \mathrm{KGW}$ laser was investigated using a micromirror and a prism in an intracavity Littman configuration, allowing tuning of the output wavelength from $1024 \mathrm{~nm}$ to $1031.5 \mathrm{~nm}$. These initial demonstrations will provide a platform towards a miniaturized solid-state laser with tunable temporal and spectral output characteristics.

\section{REFERENCES}

[1] Holmstrom, S.T.S., Baran, U. and Urey, H., “MEMS Laser Scanners: A Review,” Jour. of MEMS 23 (2), 259 275 (2014).

[2] John, D.D., Burgner, C.B., Potsaid, B., Robertson, M.E., Lee, B.K., Choi, W.J., Cable, A.E., Fujimoto, J.G. and Jayaraman, V., "Wideband Electrically Pumped 1050nm MEMS-Tunable VCSEL for Ophthalmic Imaging," Jour. of Lightwave Tech. 33 (16), 3461-3468 (2015).

[3] Fabert, M., Desfarges-Berthelemot, A., Kermène, V. and Crunteanu, A., "Temporal synchronization and spectral combining of pulses from fiber lasers Q-switched by independent MEMS micro-mirrors," Opt. Expr. 20 (20), 22895-22901 (2012).

[4] Lubeigt, W., Gomes, J., Brown, G., Kelly, A., Savitski, V., Uttamchandani, D. and Burns, D., "Control of solidstate lasers using an intra-cavity MEMS micromirror," Opt. Expr. 19 (3), 2456-2465 (2011).

[5] Bauer, R., Paterson, A., Clark, C., Uttamchandani, D. and Lubeigt, W., "Output Characteristics of Q-switched Solid-State Lasers Using Intracavity MEMS Micromirrors,” IEEE Jour. of Sel. Top. In Quant. Elec. 21 (1), $356-$ 363 (2015).

[6] Inoue, A., Komikado, T., Kinoshita, K., Hayashi, J. and Umegaki, S., "Deformable mirror for mechanical Qswitching of laser-diode-pumped microchip laser,” Jpn. Jour. Appl. Phys. 46 (42), L1016-L1018 (2007).

[7] Bauer, R., Lubeigt, W., and Uttamchandani, D., "Dual Q-switched laser outputs from a single lasing medium using an intracavity MEMS micromirror array," Opt. Lett. 37 (17), 3567-3569 (2012).

[8] MEMSCAP Inc., “a MUMPs ${ }^{\circledR}$ process,” http://www.memscapinc.com/products/mumps/soimumps.

[9] Hopcroft, M.A., Nix, W.D. and Kenny, T.W., "What is the Young's Modulus of Silicon?," Jour. of MEMS 19 (2), 229-238 (2010).

[10] Liu, K. and Littman, M.G., "Novel geometry for single-mode scanning of tunable lasers," Opt. Let. 6 (3), $117-$ 118 (1981). 